\title{
SOME REMARKS ON TIME INTEGRATION OF 3D ROTOR-STATOR ASSEMBLY
}

\author{
B. Prabel ${ }^{1}$ \\ ${ }^{1}$ CEA, DEN, DANS, DM2S, SEMT, DYN - CEA Saclay, F-91191 France \\ e-mail: benoit.prabel@cea.fr
}

Keywords: Time integrators, Rotordynamics, Model reduction, Nonlinear vibrations, FEM

\begin{abstract}
This paper focuses on the application of time integration methods to rotor-stator assembly modeled by Finite Element Method. In particular the average acceleration method (which is implicit) and the central differences method (which is explicit) are examined. Both belong to the Newmark's time integrator family and are widely used. It is found that the first one is unstable due to the periodic kinematic constraints linking the rotating to the non rotating parts of the structure. A proposed remedy is to add numerical damping leading to the implicit HHT and $\alpha$-generalized schemes. Concerning the integration with an explicit scheme, it is demonstrated that a classical implementation lead to a vanishingly small critical time step because of the strong gyroscopic effects. An exact implementation of this kind of scheme is proved to maintain the value of the critical time step.
\end{abstract}




\section{INTRODUCTION}

The need to fully control the dynamical behavior of rotors is motivated by rotors machinery more and more complex operating in more and more severe conditions. Modeling capacities are often wished to ensure the lowest cost of design and maintenance and the highest safety margins.

To this end, this work focuses on the computation of the time response of a three-Dimensional Finite Element model (3D-FE model) enabling to represent the behavior of a complex rotorstator assembly.

First, a three-Dimensional modelin the Finite Element software Cast3M [1] is considered, following the work of Combescure and Lazarus [2]. As pointed out by Geradin and Kill [3] and Chatelet et al. [4], compared to one-dimensional models (1D models), 3D-FE models exhibit better accuracy for evaluating stiffness of disc and wheel-shaft assembly. They are also required when dealing with non-axisymmetric geometry like bladed disc. They however imply an higher computational cost and some reduction techniques are necessary in industrial cases.

Time integration methods is widely viewed as the more direct manner to predict the dynamic response of a mechanical structure to a given loading in presence of some non-linearities. This general fact remains true in rotordynamics as pointed out by Genta [5] Alternatives in the frequency domain (like the Harmonic Balance Method for example) exist but their use is not always straightforward.

Hence, the present paper aims at presenting the specificity of time-integration methods when applied to a 3D rotor-stator assembly, exhibiting some little known numerical instabilities and their possible remedies.

\section{MODELING 3D ROTOR-STATOR ASSEMBLY}

\subsection{Equation of motion of a 3D rotor-stator assembly}

The more "natural" approach to model the vibrations of a rotor discretized by non-beamelement is to consider its motion in the rotating frame $\mathcal{R}^{\prime}=\left\{O^{\prime}, \underline{e}_{1}^{\prime}, \underline{e}_{2}^{\prime}, \underline{e}_{3}^{\prime}\right\}$. This one is represented on figure 1. A simple rotation defined by the angular velocity $\Omega$ around the first axis $\left(\underline{e}_{1}=\underline{e}_{1}^{\prime}\right)$, links this frame to the non-rotating Galilean frame $\mathcal{R}$.

The position and displacement of a point $P$ defined in the rotating frame by its relative coordinate can be expressed in the inertial frame by the rotation of angle $\underline{\Omega} t$ and the translation of the origin $\underline{s}=\underline{O O^{\prime}}$. Following Argyris [6] for example, the position $\underline{y}$ of $P$ is given by the coordinate $x+u$ in $\mathcal{R}^{\prime}$, and the coordinate $y$ of this point in $\mathcal{R}$ are expressed by:

$$
\begin{aligned}
& \underline{y}=\underline{O P}=\underline{O O^{\prime}}+\underline{O^{\prime} P} \\
& y=s+[R](x+u)
\end{aligned}
$$

where $[R]=\left[\begin{array}{lll}\underline{e}_{1}^{\prime} & \underline{e}_{2}^{\prime} & \underline{e}_{3}^{\prime}\end{array}\right]$ is the orthonormal matrix of the basis vectors of $\mathcal{R}^{\prime}$ expressed in $\mathcal{R}$, and $s$ is the coordinate of $\underline{O O^{\prime}}$ in $\mathcal{R}$.

To deal with vibrations, velocity and acceleration have to be computed. The derivation of basis vector is simply:

$$
\underline{\dot{e}}_{i}^{\prime}=\underline{\Omega} \wedge \underline{e}_{i}^{\prime}
$$

Then velocity and acceleration in the inertial frame can be derived as:

$$
\begin{aligned}
& \underline{\dot{y}}=\underline{\dot{s}}+\underline{\dot{x}}+\underline{\dot{u}} \\
& \dot{y}=\dot{s}+[R] \dot{u}+\underline{\Omega} \wedge([R](x+u))
\end{aligned}
$$




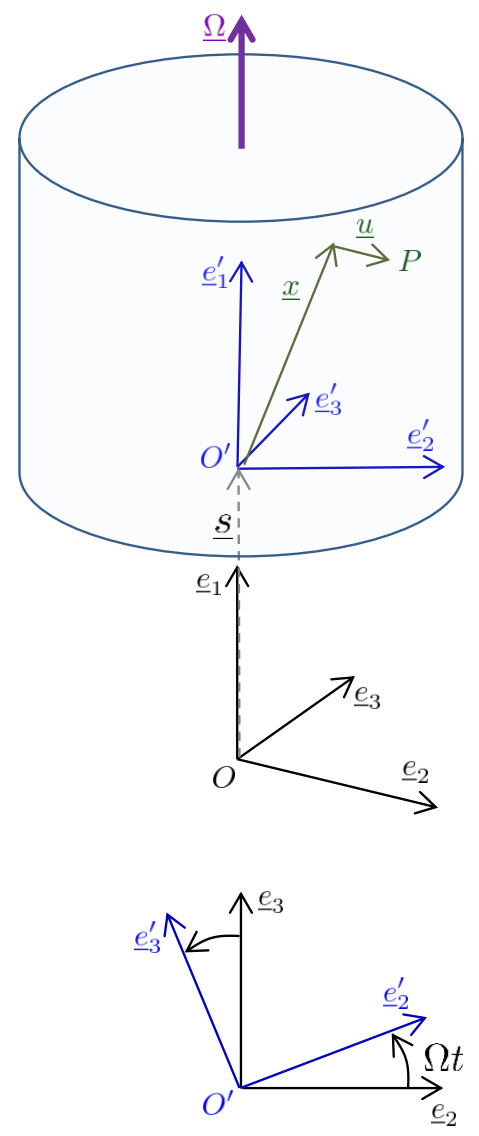

Figure 1: Definition of non-rotating (also called inertial) and rotating frames

$$
\begin{aligned}
\ddot{y} & =\ddot{s}+[R] \ddot{u}+\underline{\dot{\Omega}} \wedge([R](x+u))+\underline{\Omega} \wedge \underline{\Omega} \wedge([R](x+u))+2 \underline{\Omega} \wedge([R] \dot{u}) \\
{[R]^{T} \ddot{y} } & =[R]^{T} \ddot{s}+\ddot{u}+\dot{\Omega} \wedge(x+u)+\Omega \wedge \Omega \wedge(x+u)+2 \Omega \wedge \dot{u}
\end{aligned}
$$

The third term of the Right-Hand-side of Eq. (3b) is the Euler term and is zero as long as the spin speed is constant. The fourth term refers to the centrifugal effect acting in the radial direction. The last therm of the RHS is the probably the most characteristic of Rotordynamics as it refers to the Coriolis effect which produces a pseudo-acceleration in a direction perpendicular to the relative velocity.

The geometry of the rotor is discretized in the rotating frame with standard finite element shape functions:

$$
\underline{x}=\sum_{i} N_{i}(x) \underline{x}_{i}
$$

Isogeometric element are considered and displacements, velocity and acceleration are approximated in the same space of functions:

$$
\begin{aligned}
& \underline{u}(\underline{x}, t)=\sum_{i} N_{i}(x) \underline{u}_{i}(t) \\
& \underline{\dot{u}}(\underline{x}, t)=\sum_{i} N_{i}(x) \underline{\dot{u}}_{i}(t) \\
& \underline{u}(\underline{x}, t)=\sum_{i} N_{i}(x) \underline{\ddot{u}}_{i}(t)
\end{aligned}
$$


The weak form of the mechanical problem for the domain $\mathcal{D}$ of boundary $\partial \mathcal{D}=\partial \mathcal{D}_{F} \cup \partial \mathcal{D}_{u}$ is given by Eq. (6).

$$
\begin{aligned}
& \forall \delta \underline{v} \in V^{0}=\left\{\delta \underline{v}, \text { so that } \delta \underline{v}=0 \operatorname{sur} \partial \mathcal{D}_{u}\right\}, \\
& \int_{\mathcal{D}} \rho \underline{\ddot{u}} \cdot \delta \underline{v} d \mathcal{D}+\int_{\mathcal{D}} \underline{\underline{\sigma}}: \delta \underline{\underline{e}}(\underline{v}) d \mathcal{D}=\int_{\mathcal{D}} \underline{f}_{d} \cdot \delta \underline{v} d \mathcal{D}+\int_{\partial \mathcal{D}_{F}} \underline{F}_{d} \cdot \delta \underline{v} d \Gamma
\end{aligned}
$$

Injecting the kinematic of the rotor in the weak form of the problem, supposing that equilibrium due to centrifugal forces is verified by a static motion generating a constant state of stress $\sigma^{0}$, and using the discretized approximation of these quantities, dynamic equilibrium is obtained:

$$
[M] \underline{\ddot{u}}^{\prime}+\left[\Omega G+C_{\text {visc }}\right] \underline{\dot{u}}^{\prime}+\left[K_{\text {elas }}+\Omega^{2} K_{\text {cent }}+K\left(\sigma^{0}\right)\right] \underline{u}^{\prime}=F_{\text {ext }}^{\prime}
$$

where the Coriolis effect is $G$.

For the non-rotating part (i.e. the stator), the geometry and displacement are expressed in the inertial frame. Then, the discretized equations of motion reduce to :

$$
[M] \underline{\ddot{u}}+\left[C_{\text {visc }}\right] \underline{\dot{u}}+\left[K_{\text {elas }}\right] \underline{u}=F_{\text {ext }}
$$

\subsection{Linking the rotating to the non-rotating frame}

As depicted by figure 1, a simple change of frame enables to establish the constraint equation between the degrees of freedom of the rotor and ones of the stator. to equal their relative displacements :

$$
\begin{aligned}
& \left(\begin{array}{l}
U 1 \\
U 2 \\
U 3
\end{array}\right)=\left[\begin{array}{ccc}
1 & 0 & 0 \\
0 & \cos \Omega t & -\sin \Omega t \\
0 & \sin \Omega t & \cos \Omega t
\end{array}\right] \cdot\left(\begin{array}{l}
U 1^{\prime} \\
U 2^{\prime} \\
U 3^{\prime}
\end{array}\right) \\
& U=R(\Omega t) \cdot U^{\prime}
\end{aligned}
$$

A Lagrange multiplier $\Lambda$ is introduced to recast the constraint :

$$
\begin{aligned}
& \left(\begin{array}{ccc}
0 & -I & R \\
-I^{T} & 0 & 0 \\
R^{T} & 0 & 0
\end{array}\right) \cdot\left[\begin{array}{c}
\Lambda \\
U \\
U^{\prime}
\end{array}\right]=\left(\begin{array}{l}
0 \\
0 \\
0
\end{array}\right) \\
& \text { soit } L(\Omega t) \quad \cdot q=0
\end{aligned}
$$

\section{NEWMARK TIME INTEGRATION SCHEMES}

\subsection{Basic equations of the Newmark scheme}

A large number of literature exists about time integration scheme and the Newmark's scheme that seems to be one of the most used in structural dynamic community (see the books of Hughes [7], Géradin [8], Argyris [6] or Zienkiewicz [9] for example). It is often presented as a truncated Taylor development of local quantities which is not presented here for sake of clarity.

A recurrence formula links displacement, velocity and acceleration at time $t_{n+1}$ :

$$
\begin{aligned}
& \ddot{u}_{n+1}=\frac{1}{\beta \Delta t^{2}}\left(u_{n+1}-u_{n}\right)-\frac{1}{\beta \Delta t} \dot{u}_{n}+\left(1-\frac{1}{2 \beta}\right) \ddot{u}_{n} \\
& \dot{u}_{n+1}=\frac{\gamma}{\beta \Delta t}\left(u_{n+1}-u_{n}\right)+\left(1-\frac{\gamma}{\beta}\right) \dot{u}_{n}+\Delta t\left(1-\frac{\gamma}{2 \beta}\right) \ddot{u}_{n}
\end{aligned}
$$


Introducing these expressions in the dynamic equilibrium, one gets :

$$
\begin{aligned}
{\left[\frac{1}{\beta \Delta t^{2}} M+\frac{\gamma}{\beta \Delta t} C+K\right]\left(u_{n+1}-u_{n}\right)=F_{n+1}^{e x t} } & -K u_{n} \\
& -C\left(\left(1-\frac{\gamma}{\beta}\right) \dot{u}_{n}+\Delta t\left(1-\frac{\gamma}{2 \beta}\right) \ddot{u}_{n}\right) \\
& -M\left(-\frac{1}{\beta \Delta t} \dot{u}_{n}+\left(1-\frac{1}{2 \beta}\right) \ddot{u}_{n}\right)
\end{aligned}
$$

\subsection{Properties of the Newmark scheme}

The accuracy and stability of the Newmark scheme depend on the value of the parameters $\gamma$ and $\beta$. Table 1 sums up the stability conditions of some different schemes for a 1-dof system subjected to no external loading.

\begin{tabular}{|c|c|c|c|}
\hline Scheme & $\gamma$ & $\beta$ & $\Delta t_{c} / \Delta t^{e}$ \\
\hline Central difference & $1 / 2$ & 0 & 2 \\
Fox and Goodwin & $1 / 2$ & $1 / 12$ & 2.45 \\
Linear acceleration & $1 / 2$ & $1 / 6$ & 3.46 \\
Average acceleration & $1 / 2$ & $1 / 4$ & $\infty$ \\
Modified average acceleration & $1 / 2+\alpha$ & $1 / 4(1+\alpha)^{2}$ & $\infty$ \\
\hline Runge-Kutta 4 & - & - & $2 \sqrt{2}$ \\
Fu-De Vogelaere & - & - & $2 \sqrt{2}$ \\
\hline
\end{tabular}

Table 1: Critical time step of some time integration schemes

where $\Delta t^{e}=\frac{\min \left(\Delta x^{e}\right)}{\sqrt{E / \rho}}$ is the caracteristic time of an element of Young modulus $E$, density $\rho$ and internodal distance $\Delta x^{e}$.

The application of the central difference method to rotordynamic will be presented in the next section. Then, the average acceleration method will be considered in section $§ 5$.

\section{AN EXPLICIT SCHEME : THE CENTRAL DIFFERENCE METHOD}

\subsection{Classical approximation of the central difference method}

Choosing $\gamma=\frac{1}{2}, \beta=0$, the displacement at time $n+1$ only depends on quantities at time $n$ :

$$
u_{n+1}=u_{n}+\Delta t \dot{u}_{n}+\frac{\Delta t^{2}}{2} \ddot{u}_{n}
$$

Introducing the velocity at the mid step :

$$
\dot{u}_{n+\frac{1}{2}}=\frac{u_{n+1}-u_{n}}{\Delta t}
$$

which enables to write :

$$
\dot{u}_{n+1}=\frac{\Delta t}{2} \ddot{u}_{n+1}+\dot{u}_{n+\frac{1}{2}}
$$


the equation of motion to solve at each time step is simply :

$$
\left[M+\frac{1}{2}\right] C \ddot{u}_{n+1}=F_{n+1}^{e x t}-K u_{n+1}-C \dot{u}_{n+\frac{1}{2}}
$$

A very common practice in code implementation is to neglect the term $\frac{\Delta t}{2} C$ on the LeftHand-Side of equation (16). This leads to the "classical" central difference scheme. This assumption is generally justified by the relatively low effect of damping compared to the inertial one and to the stiffness. Combined to a lumping technique, it has the advantage to keep the operator diagonal, so that no matrix has to be inverted.

However in the case of rotordynamics, the matrix $C$ includes viscous and gyroscopic (Coriolis matrix) effects. That is why it is proposed here to consider the "Full" central difference scheme which does not neglect the participation of this term and compute the inverse of $\left[M+\frac{\Delta t}{2} C\right]$. This strategy is adopted only in the case where the spatial description is reduced by modal approach.

The effect on the performance of the scheme is evaluated on the basis of the following example.

\subsection{Application to a rotor modeled by $3 \mathrm{D} \mathrm{FE}$}

A rotor constituted of a shaft and four short blades is considered (see figure 2.

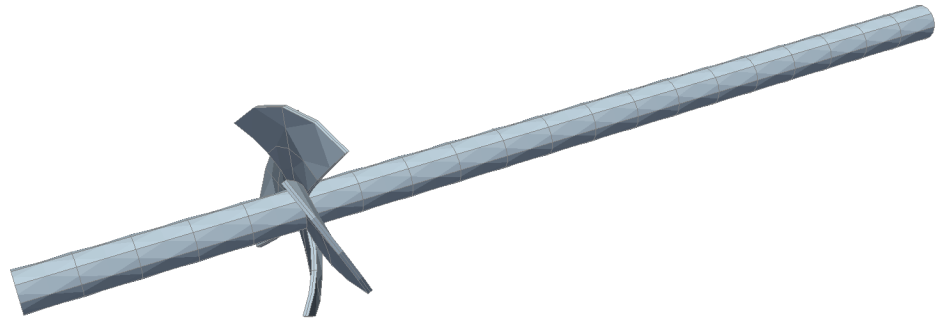

Figure 2: 3D mesh of the rotor test case

An unbalance excitation generated by a blade loss is simulating by applying an equivalent force to the model. Displacement evolution with time for different time step $\Delta t$ are given figure 3 a.

Figure 3a demonstrates that the application of the "Full" central difference scheme maintain the critical time step to an acceptable value, whereas the "classical" assumption that neglect $C$ on the LHS drastically decrease it and causes unstable behavior for $\Delta t \geq \frac{T}{256}$ (where $T=$ $2 \pi / \Omega)$. 


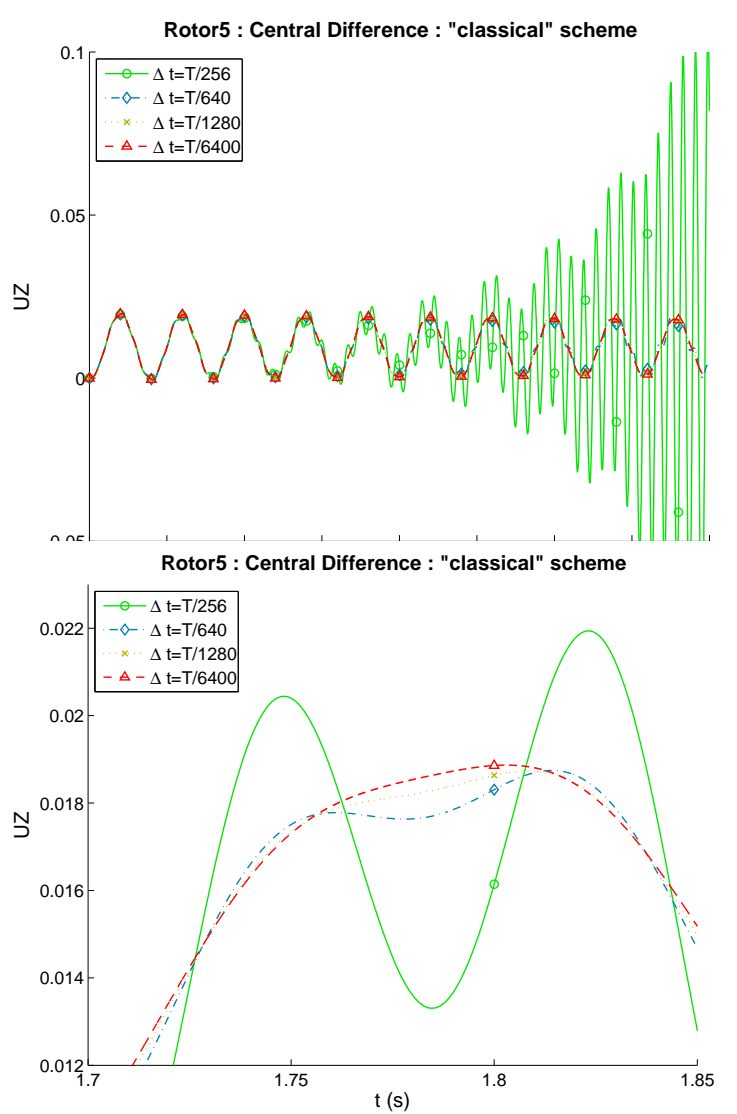

(a) "Classical" central difference scheme

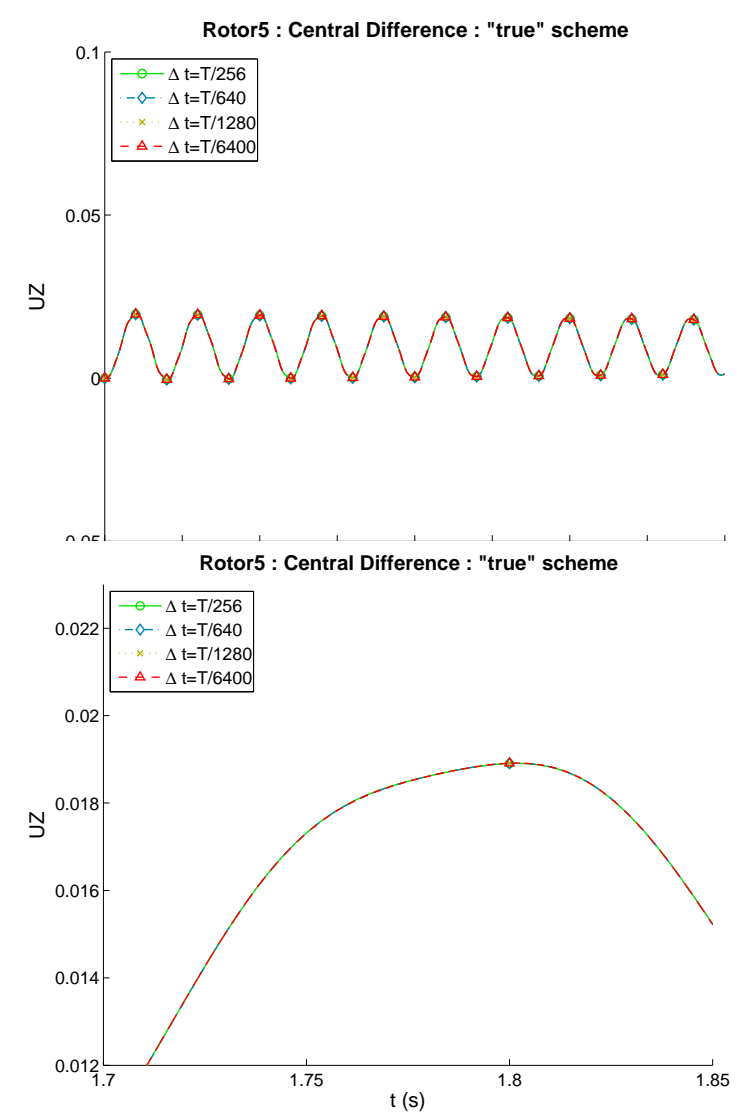

(b) "Full" central difference scheme

Figure 3: Displacement evolution with time for various time step and the explicit central difference method

\section{AN IMPLICIT SCHEME : THE AVERAGE ACCELERATION METHOD}

\subsection{Application to a 4 degree of freedom problem}

A very simple model with 4 degree of freedom is presented here to illustrate the behavior of the average acceleration scheme when used to compute the response of a rotor-stator assembly. The model is depicted on figure 4.

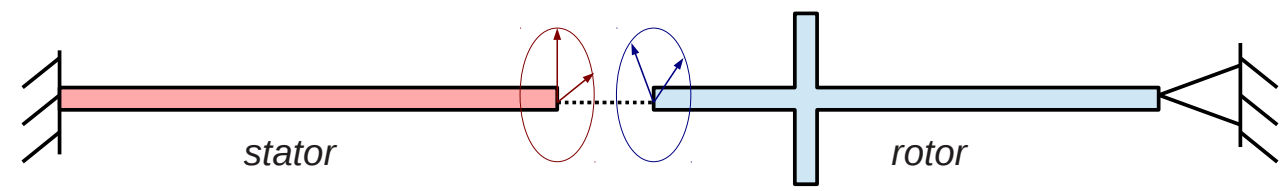

Figure 4: 4 dof rotor-stator system

Only the first modes are considered for the rotor and the stator (respectively the first flexural modes along $y$ and $z$ and the rigid body mode along $y^{\prime}$ and $z^{\prime}$ ). The introduction of the two kinematic constraints linking rotating to non-rotating parts adds two Lagrange multipliers to the system. Hence, the vector of unknown is :

$$
q^{T}=\left(\begin{array}{llllll}
\lambda_{y} & \lambda_{z} & u_{y} & u_{z} & u_{y}^{\prime} & u_{z}^{\prime}
\end{array}\right)
$$


and the equation of motion is :

$$
\begin{aligned}
& K q+C \dot{q}+M \ddot{q}=F \\
& \text { with } K=\left[\begin{array}{cccccc}
0 & 0 & 1 & 0 & -\cos \Omega t & \sin \Omega t \\
& 0 & 0 & 1 & -\sin \Omega t & -\cos \Omega t \\
& & k & 0 & 0 & 0 \\
& & & k & 0 & 0 \\
& & & & k^{\prime} & 0 \\
\text { sym } & & & & & k^{\prime}
\end{array}\right] \quad C=\left[\begin{array}{cccccc}
0 & 0 & 0 & 0 & 0 & 0 \\
& 0 & 0 & 0 & 0 & 0 \\
& & c & 0 & 0 & 0 \\
& & & c & 0 & 0 \\
& & & & c^{\prime} & 0 \\
\text { sym } & & & & & c^{\prime}
\end{array}\right] \\
& \text { and } M=\left[\begin{array}{cccccc}
0 & 0 & 0 & 0 & 0 & 0 \\
& 0 & 0 & 0 & 0 & 0 \\
& & m & 0 & 0 & 0 \\
& & & m & 0 & 0 \\
& & & & m^{\prime} & 0 \\
\text { sym } & & & & & m^{\prime}
\end{array}\right]
\end{aligned}
$$

An unbalance loading is applied to the rotor along the $y^{\prime}$-direction :

$$
F=(2 \pi \Omega)^{2} \cdot r(t) \cdot\left(\begin{array}{llllll}
0 & 0 & 0 & 0 & f & 0
\end{array}\right)^{T}
$$

with a linear ramp variation during the half of the first period :

$$
r(t)= \begin{cases}2 \Omega t & \text { if } \quad 0 \leq t<\frac{1}{2 \Omega} \\ 1 & \text { else }\end{cases}
$$

Numerical values used in for this test case are given table 2.

\begin{tabular}{|c|c|c|c|c|c|c|c|}
\hline $\mathrm{m}$ & m' & $\mathrm{k}$ & $\mathrm{k}$ & $\mathrm{c}$ & $\mathrm{c}^{\prime}$ & $\mathrm{f}$ & $\Omega$ \\
\hline 1E3 & $1 \mathrm{E} 3$ & $6 \mathrm{E} 6$ & 0 & $31 \mathrm{E} 3$ & 0 & $\frac{1}{(2 \pi \Omega)^{2}}$ & $5 \mathrm{~Hz}$ \\
\hline
\end{tabular}

Table 2: Numerical value of the 4-dof system

Solving equation (19) with the average acceleration method lead to the results of figure 5.

As there is no physical source of instability in the proposed model, the simple 4-dof example presented here proved the instability of the Newmark average acceleration method for this class of linear but with periodic constraints problem! This is contradictory with the unconditionally stable reputation of this scheme for linear problem. Evolution of Lagrange multipliers suggests that the periodic constraint is the source of numerical divergence of the solution.

A single article of 1989 written by Cardona and Géradin [12] demonstrates that some kinematics constraints may lead to infinite eigenvalues of the amplification matrix. Time integration of systems with a relative important participation of kinematic constraints is then not an easy task. This explains why a great amount of literature can be found about time-integration in flexible multibody dynamics [13].

Three solutions can be adopted to treat this problem :

- When possible, eliminate the constraints (and the associated variables),

- Derive two times the constraint equation and add mass and damping terms so that the constraint becomes an overdamped system, 


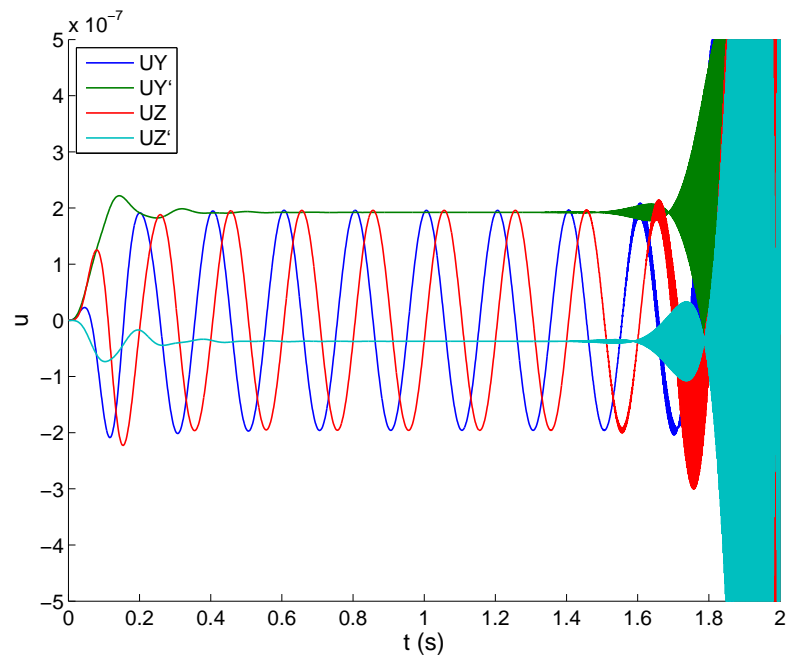

(a) Displacement time evolution up to $2 \mathrm{~s}$

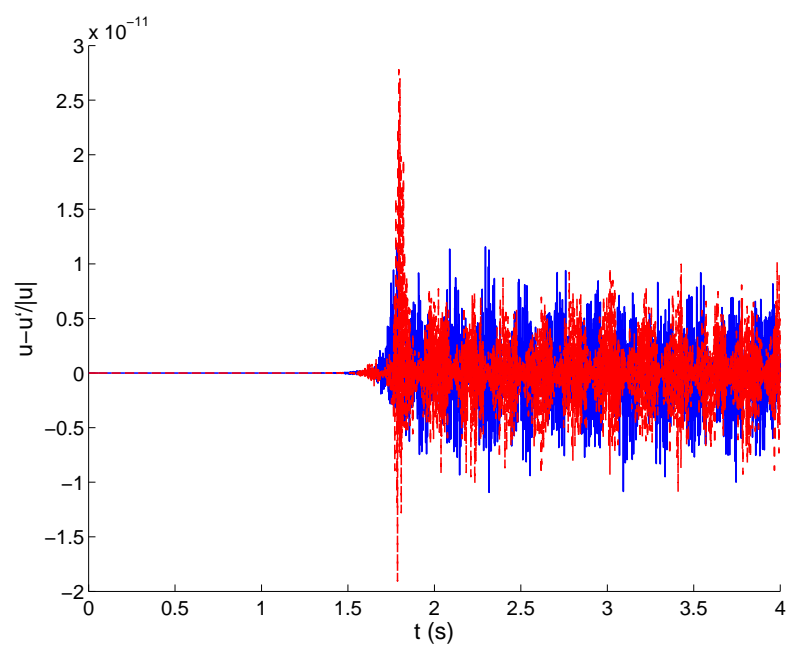

(c) Relative validity of the constraint rotor-stator

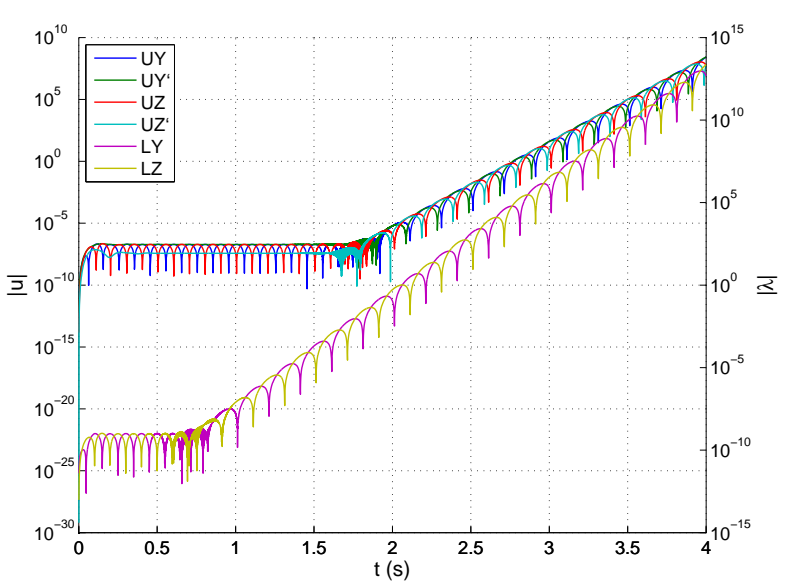

(b) Lagrange multipliers time evolution

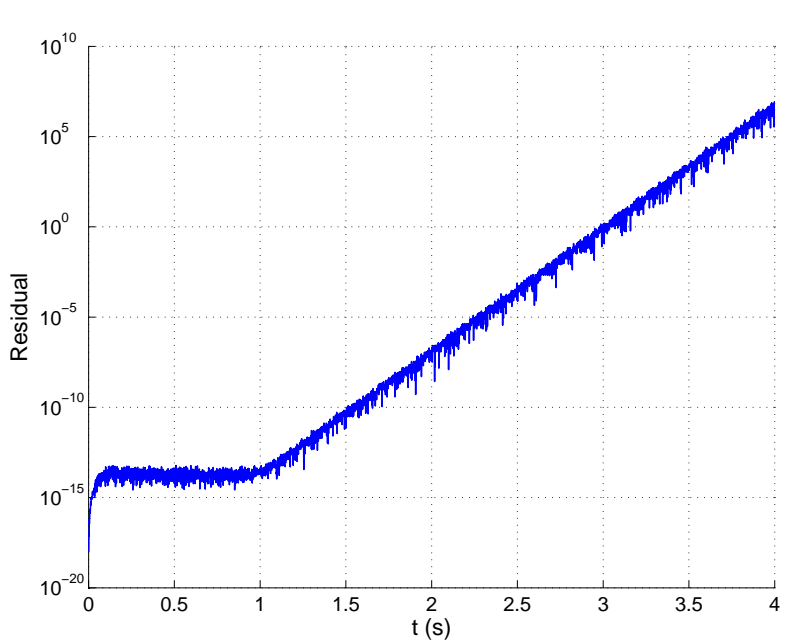

(d) Evolution of the residual of the dynamic equilibrium

Figure 5: Results of the average acceleration method for the 4-dof system

- Use a scheme which presents naturally numerical damping.

The last one will be discussed in the following subsection. 


\subsection{Adding numerical damping to the scheme}

Three scheme with numerical damping have been implemented in Cast $3 \mathrm{M}$ and tested on the 4-dof test case :

- Modified average acceleration with $\alpha=0.05$

- HHT with $\alpha=0.05$

- $\alpha$-generalized with $\rho_{\infty}=0.9048$

The spectral radius (identified as the highest eigenvalue of the amplification matrix) and the numerical damping are plotted for these scheme figure 6 . Figure 6 shows that high frequencies

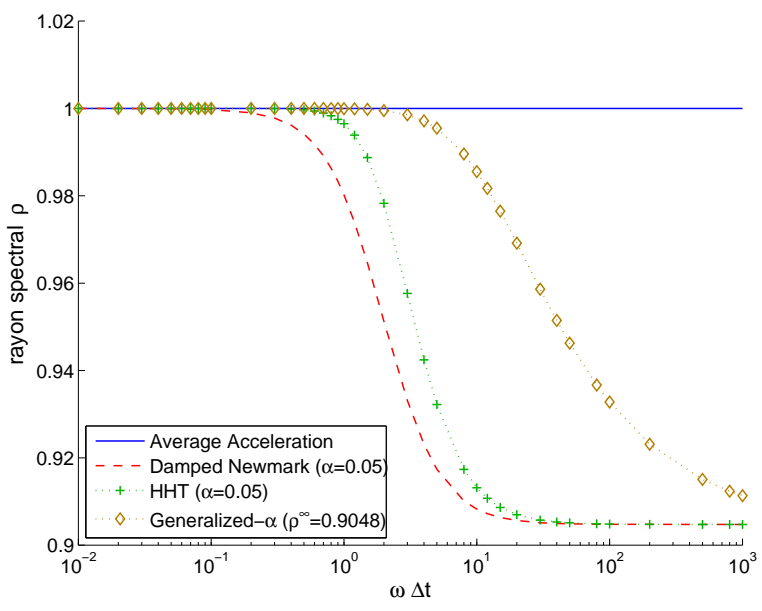

(a) Spectral raidus

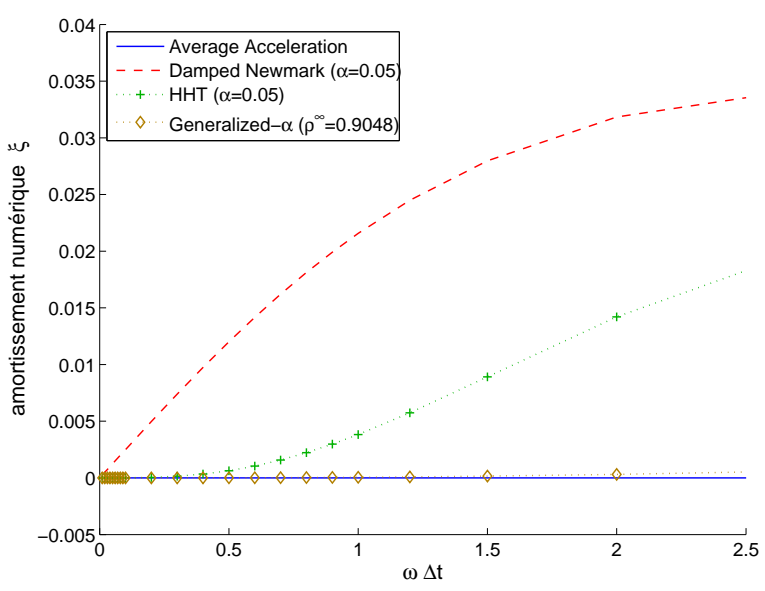

(b) Damping

Figure 6: Characteristics of some schemes

are considered as spurious and attenuated by these schemes.

The HHT (from Hilber-Hughes-Taylor [10]) and the $\alpha$-generalized [11] methods are methods closed to the Newmark's one, but a weighting of elastic, viscous and inertial forces over time step is performed in order to control the damping. The general equation of motion to solve is no more (8) but :

$$
\begin{aligned}
\left(1-\alpha_{m}\right) M \ddot{u}_{n+1}+\alpha_{m} M \ddot{u}_{n}+\left(1-\alpha_{f}\right) C \dot{u}_{n+1}+\alpha_{f} C \dot{u}_{n}+ & \left(1-\alpha_{f}\right) K u_{n+1}+\alpha_{f} K u_{n} \\
& =\left(1-\alpha_{f}\right) F_{n+1}^{e x t}+\alpha_{f} F_{n}^{e x t}
\end{aligned}
$$

where $\alpha_{m}=0$ for the HHT method. The Newmark parameters are adjusted to maintain second order accuracy :

$$
\gamma=\frac{1}{2}+\alpha_{f}-\alpha_{m} \quad \text { et } \quad \beta=\frac{1}{4}\left(1+\alpha_{f}-\alpha_{m}\right)^{2}
$$

For the $\alpha$-generalized method, the spectral radius $\rho_{\infty} \omega \Delta t \rightarrow \infty$ can be chosen and the coefficients $\alpha_{m}$ and $\alpha_{f}$ deduced as :

$$
\alpha_{m}=\frac{2 \rho_{\infty}-1}{\rho_{\infty}+1} \quad \text { et } \quad \alpha_{f}=\frac{\rho_{\infty}}{\rho_{\infty}+1}
$$

Using one of the three aforementioned scheme leads to a slightly damped stable solution as depicted by figure $7 \mathrm{a}$. 


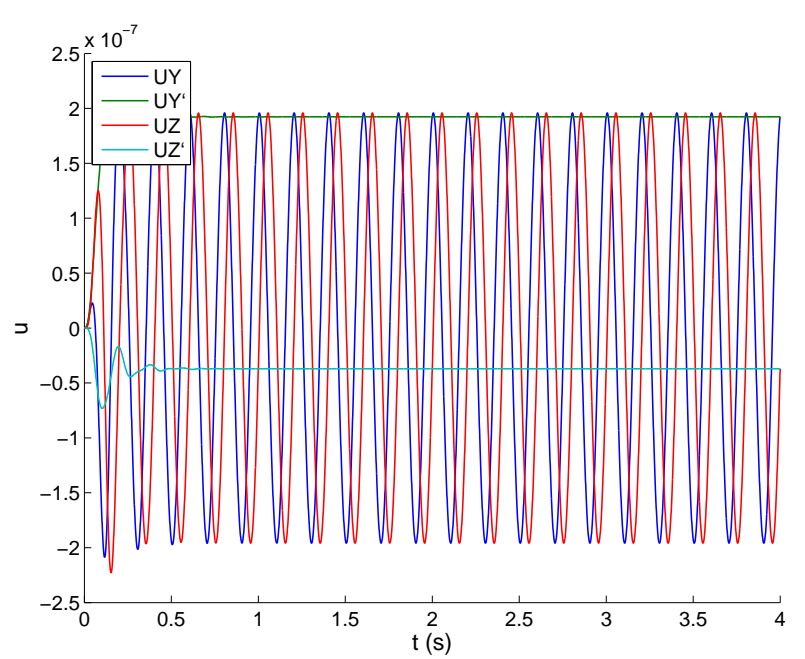

(a) Time evolution of displacements obtained with the HHT scheme $(\alpha=0.05)$

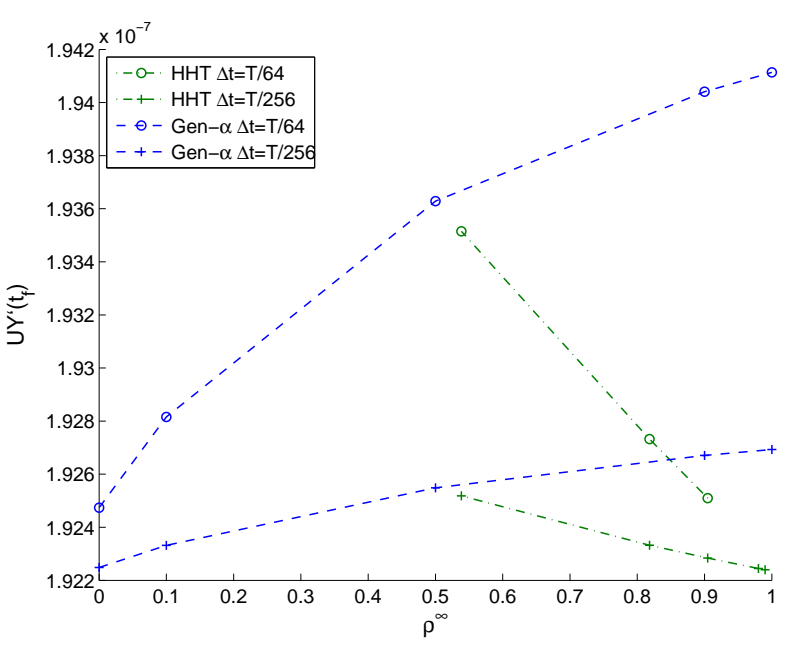

(b) Displacement UY' at th eend of simulation

Figure 7: Results obtained with numerical damping

The stationary value of the UY' displacement as a function of the spectral radius for the HHT and $\alpha$-generalized methods and for two values of time step is given figure $7 \mathrm{~b}$. For this very simple 4-dof system, the analytic solution at steady state is $U^{\prime} Y^{\prime}=1.922 \mathrm{E}-7$. Observation of this figure indicates that :

- with the HHT scheme, instability is observed for too small values of $\alpha_{f}$ and this critical value depends on time step,

- with the HHT scheme, the amplitude of vibration tends to decrease with the spectral radius, which is counter-intuitive !

- with the $\alpha$-generalized scheme, the system is always stable even if $\rho^{\infty}=1$ (no dissipation)!

- with the $\alpha$-generalized scheme, the finale amplitude increase with the spectral radius but remains a finite value,

- a better solution is found for smaller time step and for respectively large and small radius for $\mathrm{HHt}$ and $\alpha$-generalized schemes.

\subsection{Application to 3D rotor-stator assembly}

A system very close to the 4-dof previously studied system is considered. The 3D FE mesh of this system is given figure 8 .

Average acceleration method is first used with two spatial discretization :

- FE dof : the Finite Element degree of freedom,

- CMS dof : a Component Mode Synthesis method (Craigh Brampton) is used to reduce the number of dof.

Mode shapes used in CMS are plotted figure 9. 


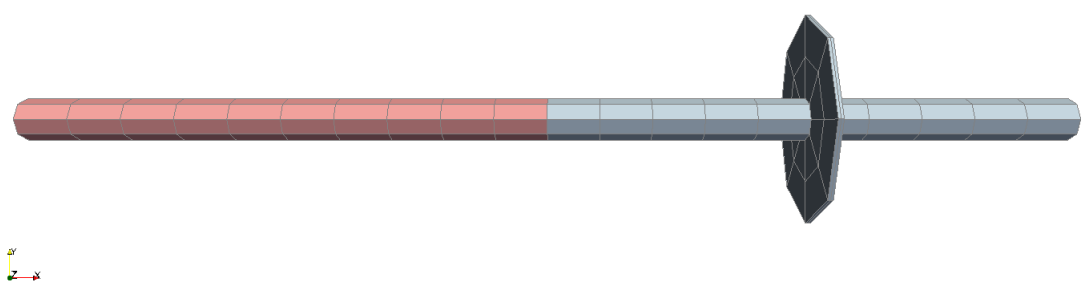

Figure 8: FE mesh : the stator is on the left (pink) and the rotor is on the right (light blue)

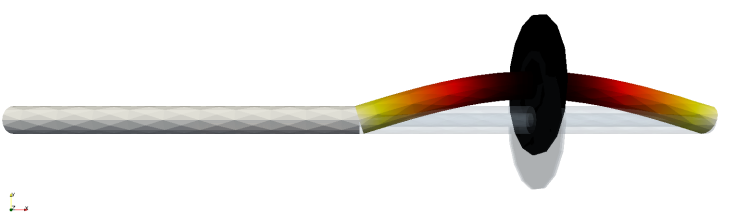

(a) Eigenmode (first flexion of the rotor)

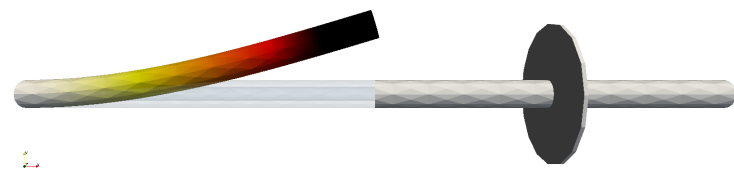

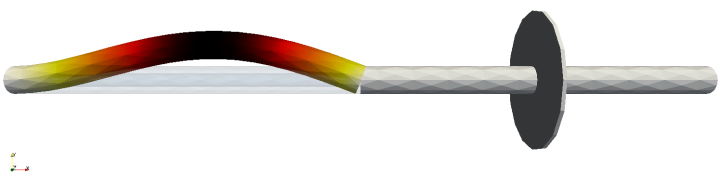

(b) Eigenmode (first flexion of the stator)

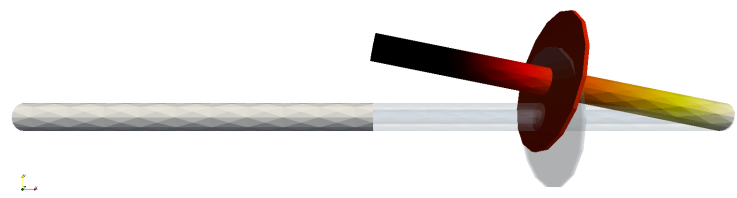

(c) Static solution (lateral displacement imposed to the(d) Static solution (lateral displacement imposed to the rotor) stator)

Figure 9: Mode shape constituting the CMS base

The displacement evolution with time for both spatial discretization is given figure ?? Whereas FE-dof based response remains stable, it seems that CMS-dof based solution become unstable like the 4-dof system.

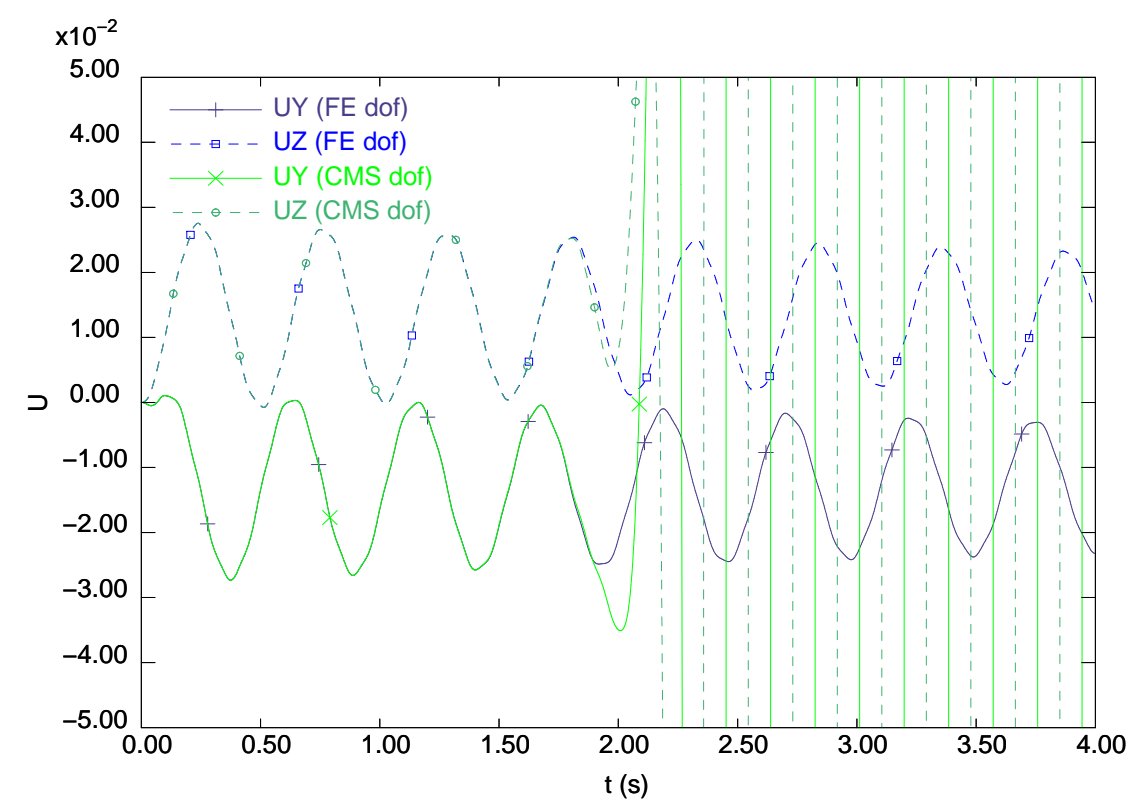

transitoire $(\Omega=5 \mathrm{~Hz})$ - DYNAMOI physique: acc moy. - modal: $\mathrm{HHT} \alpha_{\mathrm{F}}=0$

Application of the numerically damped HHT and $\alpha$-generalized methods enables to get ride 
of this instability as it is shown on figures 10 .
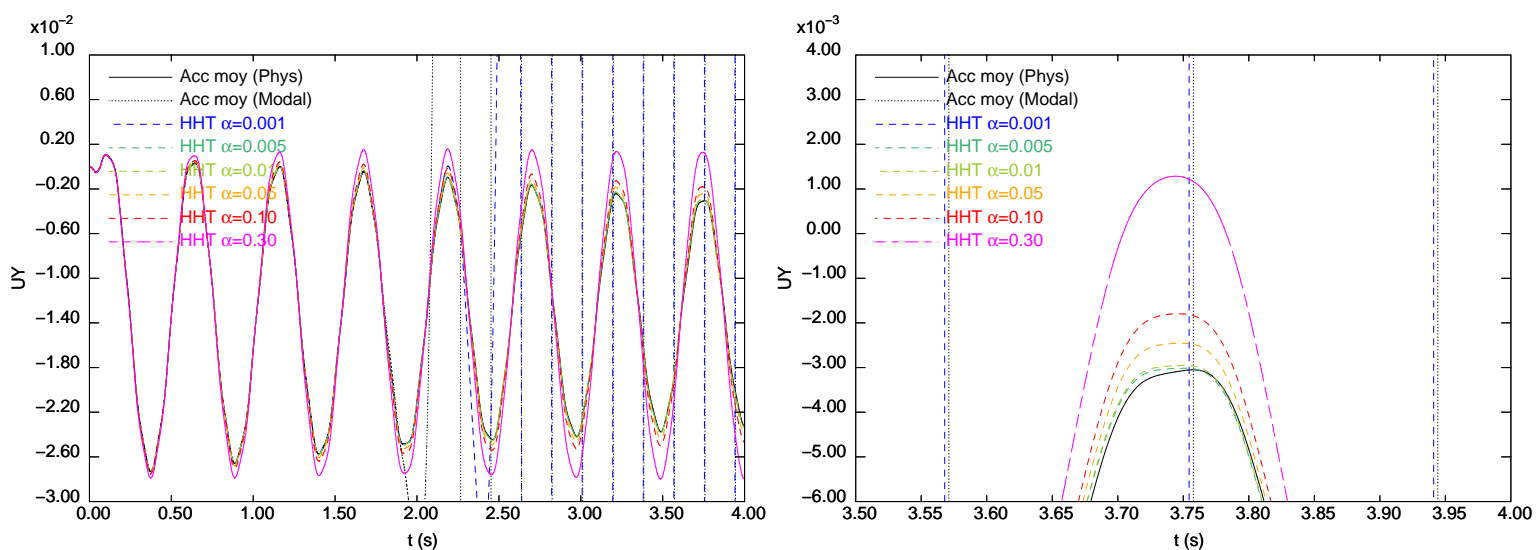

rotorstator5 3D : comparaison algorithme

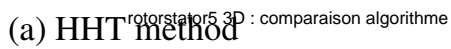
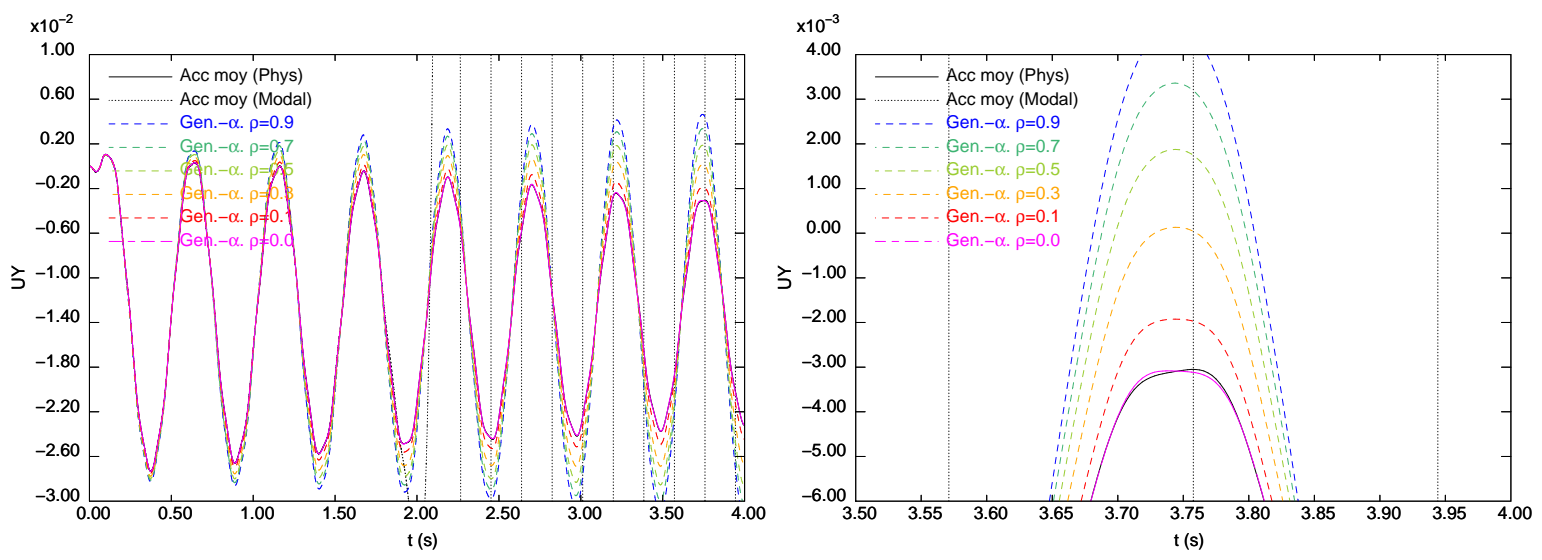

rotorstator5 3D : comparaison algorithme

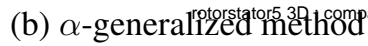

Figure 10: Results of time integration applied to 3D rotor-stator assembly

Once again, the HHT method seems efficient for a "just stable" value of parameter $\alpha_{f}=$ 0.005 , whereas the $\alpha$-generalized method is the closest to the reference for an high damping value $\left(\rho^{\infty}=0\right)$.

\section{CONCLUSIONS AND PERSPECTIVES}

Two kinds of numerical instabilities have been highlighted for both the central difference and the average acceleration method.

The first one concerns the drop of the critical time step when neglecting the gyroscopic term in the operator computing the acceleration at the next time step. A simple remedy is to use the full formulation but it could be expensive if the inversion of a large matrix have to be performed.

For implicit scheme, matrix inversion is naturally done and the time step is expected to be greater. However the kinematic constraints to link the rotating to the non-rotating parts introduce a numerical instability which has to be dissipated. HHT and $\alpha$-generalized methods have been found to do this task under the condition to choose appropriately the numerical damping.

As this choice may not be obvious in every situations, combination of an explicit integrator with a component mode synthesis method seems to be the most attractive way to model complex $3 \mathrm{D}$ rotor-stator assembly. 
Future development will concern the integration of nonlinear component (bearing, squeezefilm, rotor-stator contact, ...) in the models.

\section{REFERENCES}

[1] Cast3M Finite Element software, CEA, http: //www-cast 3m. cea.fr, 2015.

[2] D. Combescure, A. Lazarus, Refined finite element modelling for the vibration analysis of large rotating machines: Application to the gas turbine modular helium reactor power conversion unit. Journal of Sound and Vibration, 318(4), 1262-1280, 2008.

[3] M. Geradin, N. Kill, A new approach to finite element modelling of flexible rotors. Engineering Computations, 1(1), 52-64, 1984.

[4] E. Chatelet, F. D?Ambrosio, G. Jacquet-Richardet, Toward global modelling approaches for dynamic analyses of rotating assemblies of turbomachines. Journal of Sound and Vibration, 282(1), 163-178, 2005.

[5] G. Genta, Dynamics of rotating systems. Springer, 2005.

[6] J. H. Argyris and H.-P. Mlejnek, Dynamics of structures. North-Holland Amsterdam, 1991.

[7] T. J. Hughes, The Finite Element Method: Linear Static and Dynamic - Finite Element Analysis. Prentice-Hall International, Inc., 1987.

[8] M. Géradin and D. Rixen, Théorie des vibrations: application à la dynamique des structures. Masson Paris, 1993.

[9] O. Zienkiewicz, R. Taylor, and J. Zhu, The Finite Element Method: Its Basis And Fundamentals. Butterworth-Heinemann, 6th edition ed., 2005.

[10] H. M. Hilber, T. J. Hughes, and R. L. Taylor, Improved numerical dissipation for time integration algorithms in structural dynamics, Earthquake Engineering \& Structural Dynamics, vol. 5, no. 3, pp. 283-292, 1977.

[11] J. Chung and G. Hulbert, A time integration algorithm for structural dynamics with improved numerical dissipation: the generalized- $\alpha$ method, Journal of applied mechanics, vol. 60 , no. 2 , pp. 371-375, 1993.

[12] A. Cardona and M. Géradin, Time integration of the equations of motion in mechanism analysis, Computers \& Structures, vol. 33, no. 3, pp. 801-820, 1989.

[13] O. A. Bauchau, Flexible multibody dynamics, vol. 176. Springer, 2010. 